\title{
CRIAÇÃO DE UM MODELO DE CLASSIFICAÇÃO DE TWEETS EM PORTUGUÊS DO BRASIL RELACIONADOS A CRIMES UTILIZANDO MÁQUINA DE VETORES DE SUPORTE
}

\author{
Isa Cristina C. de Andrade, Áurea Milene Teixeira B. dos Santos, Jonatã P. da Costa \\ e Roberto C. Limão de Oliveira \\ Universidade Federal do Pará, (UFPA), Caixa Postal 66.075 -110-Belém-PA-Brasil
}

\begin{abstract}
RESUMO
O Twitter se tornou uma grande fonte de dados de pesquisa para descoberta de conhecimento, sendo uma rede social que divulga o compartilhamento de opiniões e informações sobre eventos em geral, além de informações pessoais. Considerando isso, o presente estudo tem como objetivo desenvolver um classificador com o propósito de classificar tweets de notícias relacionadas a crime ou não escritos em português do Brasil, utilizando o método de reconhecimento de padrões SVM (Support Vector Machine - Máquina de Vetores de Suporte). O ambiente de desenvolvimento utilizado foi o Jupyter Notebook, onde a partir de várias bibliotecas auxiliares foram implementadas técnicas de pré-processamento nos tweets para eliminar informações indesejáveis. O dataset utilizado no experimento é composto por tweets de contas jornalísticas da cidade de Belém, Pará, Brasil. Os tweets são submetidos a um classificador SVM (Linear e RBF) onde o Linear obteve uma taxa de acerto de $97 \%$.
\end{abstract}

PALAVRAS-CHAVE

Twitter, SVM, Classificação, Crime

\section{INTRODUÇÃO}

Juntamente com o crescimento do acesso à Internet, é notável o crescimento do uso das redes sociais na última década. Dentre as centenas de redes sociais, o Twitter é uma plataforma consolidada existindo milhares de pessoas que o usam constantemente com a intenção de exteriorizar suas opiniões e sentimentos sobre algum tópico em forma de texto e com uma restrição de 140 caracteres. Em abril de 2019, a empresa revelou um tráfego médio diário de 134 milhões de usuários, seja publicando ou lendo publicações de outros usuários (FOLHA, 2019). O conjunto de caracteres de cada uma das publicações é usualmente denominado de tweet.

Segundo (FILHO, 2014), os usuários das redes sociais não são mais apenas consumidores de informação, mas também geradores de conteúdo, já que lhes é oferecida a oportunidade de expressar as suas opiniões por meio de postagens, comentários e discussões em fóruns. Essa disseminação de opiniões e ideias acabaram chamando a atenção de estudiosos e empresas que procuram encontrar dados relevantes dentre a imensa quantidade de informação que é gerada a cada momento na rede (BOTHOS; APOSTOLOU; MENTZAS, 2010). Dessa forma, os usuários das redes sociais não se limitaram à interação com conhecidos, mas acabaram transformando-as em espaços para se divulgar produtos e serviços, buscando informações sobre acontecimentos políticos, sociais, calamidades e tragédias em tempo real, comentar e compartilhar sobre a sua vida cotidiana, entre outras atividades.

Devido a essa enorme quantidade de publicações através de base, o Twitter se tornou uma grande fonte de informação sobre diversas questões da vida diária da sociedade mundial e diversas análises e experimentos de aprendizado de máquina (Machine Learning) têm sido utilizados para colher informações e analisar tendências. Nas eleições presidenciais americanas, por exemplo, o Twitter foi usado para monitorar a análise de sentimento dos usuários perante aos candidatos, sendo portanto, uma ferramenta estratégica importante 
para as chapas eleitorais saberem o que estava sendo repercutido de forma positiva ou negativa sobre as propostas de governo a serem implementadas (KAPKO, 2016).

Os utilizadores desta rede social expressam-se de forma clara e concisa através de mensagens curtas e objetivas em que as opiniões podem ser comentadas nas mais diversas áreas. Os dados do Twitter são particularmente interessantes, porque, são postados na velocidade em que as pessoas expressam seus pensamentos e os dados ficando disponíveis para consumo, pois, ocorre quase em tempo real. Além disso, os tweets conectam as pessoas de várias maneiras, desde diálogos curtos e significativos, (Java et al., 2007).

Textos jornalísticos são mal avaliados em pesquisas de análise de sentimento (Carvalho, 2018). Os internautas buscam entretenimento e informação, por isso é relevante observar os conteúdos jornalísticos (Massuchin \& de Sousa, 2020). Os jornais publicam notícias nas redes sociais sobre crimes e destacam homicídios, roubos, estupros. A segurança pública e a sociedade em geral têm o desafio de compreender as diferenças no desenvolvimento humano em comunidades mais e menos violentas (Cerqueira et al., 2019).

Nesta pesquisa, utilizou-se um dataset que contem tweets coletados da rede social Twitter da conta @ doldiarionline (que pertence a um jornal local da cidade de Belém, Pará, Brasil) todos em português do Brasil. Com o objetivo de treinar um classificador e categorizar se um tweet contém uma denúncia de crime, de forma binária. Este trabalho adotou o SVM (Support Vector Machine).

\section{TRABALHOS CORRELATOS}

O Twitter é uma rede social muito utilizada para esse tipo de estudo, devido ser uma grande fonte de opiniões vindas do mundo inteiro. Possuindo um público bastante heterogêneo, e a quantidade de texto publicado na plataforma aumenta a cada momento. Alguns trabalhos vêm sendo desenvolvidos no intuito de correlacionar o sentimento seja positivo ou negativo dos tweets com relação a algum acontecimento ou evento.

Abordando discurso de ódio o trabalho de (Awan, 2016), utiliza a técnica de análise de sentimentos aplicada a discursos de ódio contra comunidades muçulmanas no Facebook.

Já o trabalho de (Corrêa, 2017) realiza a criação de um modelo de classificação de tweets depressivos utilizando o método de reconhecimento de padrões SVM, obteve-se uma taxa de acerto de $99.7 \%$ com os dados de treinamento otimizados pelo método de Grade de Busca.

O desafio de usar ferramentas de Processamento de Língua Natural em inglês para textos não estruturados em outras línguas, motivou o estudo com classificadores para trabalhar análise de sentimentos com a língua Árabe (Alwakid, Osman \& Hughes-Roberts, 2017), assim como a pesquisa em tweets escritos em tailandês realizada pelos (Wunnasri, Theeramunkong e Haruechaiyasak, 2013). Sendo que este segundo trabalho abordou a análise de sentimentos em tweets escritos em tailandês, com uma base de dados desbalanceada aplicando o classificador KNN e alcançando uma média do F1-score de 57\%.

Percebeu-se que há uma certa ausência de trabalhos que analisem e realizem uma classificação em relação ao tweet ter conteúdo referente a crime ou não.

\section{MINERAÇÃO DE TEXTO}

Mineração de texto é um processo de descoberta de conhecimento, potencialmente útil e previamente desconhecida com bases de dados desestruturados, extração de conhecimento útil, para o usuário com plataformas textuais.

O principal objetivo da Mineração de Textos (MT) consiste na extração de características em uma grande quantidade de dados não estruturados. Segundo Weiss et al. (2010), as técnicas utilizadas na mineração de textos são semelhantes às utilizadas na mineração de dados, ou seja, fazem uso dos mesmos métodos de aprendizagem, independente de técnica de dados textuais (MT) ou numéricos (MD).

Podem-se diferenciar as duas técnicas a partir de dois conceitos: a Mineração de Dados é caracterizada por extrair informações implícitas, anteriormente desconhecidas, mas potencialmente úteis. Na Mineração de Textos, a informação que se deseja extrair é clara, sendo explicitada nos textos, porém o problema é que a informação não é clara de uma maneira que seja passível de processamento automático (WITTEN; FRANK, 2011). 
A mineração de texto é um processo para a obtenção de informações importantes de um texto. As ferramentas de mineração de texto põem a disponibilização um conjunto de métodos e técnicas de análise e extração de informações a partir de textos, frases ou palavras. Segundo Nayak et al. (2016, p.16876), a "mineração de texto é o processo de busca ou extração de informações úteis dos dados textuais. Ele tenta encontrar padrões interessantes de grandes bases de textos". Com isso, o propósito da mineração de texto baseia-se na recuperação de informações, extração de dados, aprendizado de máquina, estatísticas, entre outros. O objetivo da mineração de texto é de extração de conhecimentos valiosos, a partir de dados não estruturados. Segundo Irfan et al. (2015, p.158) a mineração de texto é um processo de descoberta de conhecimento usado para extrair informações importantes e não irrelevantes da linguagem natural.

\subsection{Classificação de Texto}

Um dos motivos para o crescente interesse nos estudos sobre a área da mineração de textos, especificamente na técnica de classificação, é devido ao crescimento e à disponibilidade de documentos na internet, sobretudo nas redes sociais.

A técnica dominante para este problema é baseada na aprendizagem de máquina, ou seja, um processo indutivo cria automaticamente um classificador por "aprendizado", a partir de um conjunto de dados classificados previamente. A vantagem dessa abordagem é a independência de domínio. Pode-se dizer então que a tarefa de classificar um texto automaticamente é uma derivação da aprendizagem de máquina com o propósito de atribuir rótulos pré definidos a documentos textuais (SEBASTIANI, 2002).

Sebastiani (2002) assegura que a classificação de textos consiste em determinar se um documento di, (de um conjunto de documentos D) é pertencente ou não a uma categoria cj (de um conjunto de categorias C), consistentemente com o conhecimento das categorias corretas para um conjunto de documentos de treinamento.

O objetivo principal da classificação é atribuir uma determinada classe a um conjunto de documentos. No caso da análise de sentimentos, trata-se de classificar automaticamente um conjunto de dados às classes positivas e negativas.

\subsection{SVM}

O SVM é uma técnica de Aprendizagem de Máquina desenvolvida por Vapnik em 1995, que é fundamentada na Teoria de Aprendizado Estatístico e utilizada para a classificação de dados [Lorena e Carvalho 2003]. Smola e demais autores em [Smola et al. 1999] citam que a boa capacidade de generalização e a robustez em grandes dimensões de dados são algumas das principais características do SVM que tornam o seu uso atrativo.

Originalmente o SVM foi projetado para a classificação binária de dados. Para duas classes, poder haver muitos possíveis separadores lineares. Intuitivamente, a fronteira de decisão traçada no meio do espaço vazio entre os itens de dados das duas classes parece ser melhor do que uma que se aproxima muito de exemplos de uma ou de ambas as classes. Enquanto alguns métodos de aprendizagem como o algoritmo Perceptron encontra apenas um separador linear outros, como o Naive Bayes, procura o melhor separador linear de acordo com algum critério. Em particular, o SVM define o critério que maximiza a superfície de decisão. Esta distância, a partir da superfície de decisão para o ponto mais próximo de dados, determina a margem do classificador. Este método de construção implica necessariamente que a função de decisão para uma SVM esteja completamente especificada por um subconjunto (geralmente pequeno) dos dados, que define a posição do separador. Estes pontos são referidos como os vetores de suporte [Manning et al. 2008].

É um classificador também muito utilizado por ser efetivo em classificação de texto. O mesmo é baseado na teoria de aprendizagem estatística, desenvolvida por Vapnik (2000). Em um caso de duas classes, como é o deste trabalho, a ideia básica é a de que durante o treino do classificador, é gerado um hiperplano representado por um vetor $w^{\rightarrow}$, que não só separa uma classe da outra, mas que garante que a separação/margem seja o mais larga possível. O que corresponde a um problema de otimização restrito, ou seja, $c j \in\{1,-1\}$ (onde, 1 corresponde a classe positiva e -1 negativa). A Figura 1 ilustra um exemplo de hiperplano com duas classes para classificação: 


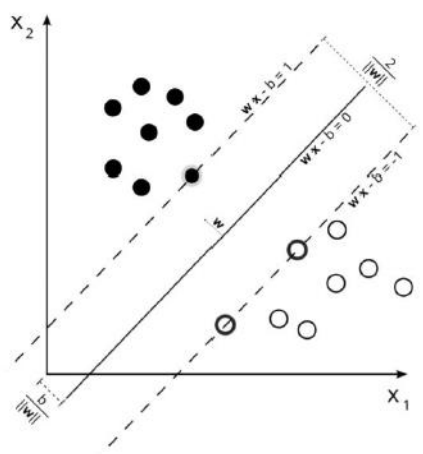

Figura 1. Exemplo do Hiperplano do SVM Fonte: Vapnik (2000)

Sendo assim, a posição de uma entrada no teste, quão próxima ou distante do ponto definido pelo treino, indica ao classificador a que classe essa entrada pertence. A equação (1) que define a teoria é a seguinte:

$$
w^{\rightarrow \rightarrow}=\Sigma \alpha j c j d j j, \geq 0
$$

Onde, $\alpha j$ 's são obtidos resolvendo o problema de otimização de duas classes, conforme Pang et al. (2002), por fim $d j$ tal que $\alpha j \geq 0$, são chamados de vetores de suporte, dessa forma o trabalho do classificador consiste em determinar de que lado do hiperplano de $w^{\rightarrow}$ a entrada de teste se encontra. A implementação escolhida para os testes foi a SVC (Serviço Virtual de Continência), disponibilizada pelo sci-kit.

É necessário descobrir a largura da distância de separação do hiperplano em relação aos dados, que pode ser dado pela diferença de dois vetores que se encontram na borda do lado positivo e do negativo, Zanchini (2019).

De acordo com Junior Gilson (2010), as máquinas de vetores suporte (support vector machines, mais conhecida como SVMs), desenvolvidas por Vapnik e colaboradores, têm a capacidade de resolver problemas de classificação e regressão.

O objetivo é produzir um classificador que funcione de forma adequada com exemplos não conhecidos, ou seja, exemplos que não foram aplicados durante o treinamento, adquirindo assim a capacidade de predizer as saídas de futuras novas entradas.

\section{METODOLOGIA}

Para o desenvolvimento desta pesquisa foi utilizada a sequência de passos comuns a mineração de texto. O primeiro passo foi a extração de um conjunto de tweets de contas jornalísticas. Os tweets foram rotulados manualmente. Posteriormente realizado o pré-processamento de dados para remover ruídos e termos irrelevantes. Realizou-se a aplicação da vetorização das palavras, ou seja, uma forma estruturada para entrada dos dados no classificador selecionado. O passo final foi a realização do treino do classificador para obter um modelo de classificação identificando os tweets sobre crimes. Este classificador poderá ser aplicado a novos tweets, sendo o modelo capaz de inferir a qual classe o texto pertence.

\subsection{Obtenção dos Datasets e Pré-Processamento dos Dados}

Para se criar um classificador de tweets, deve-se fornecer exemplos do que seriam tweets criminais e não-criminais para a etapa de treinamento. Assim, após o treinamento supervisionado, o modelo estará pronto para distinguir dados das duas classes.

Os tweets criminais e não criminais foram obtidos em (COSTA, J. P. \& Tal., 2019) a sua fonte da extração foram as principais contas jornalísticas que já publicam no jornal impresso, televisivo ou rádio sobre 
os crimes que acontecem na cidade de Belém, Pará, Brasil. A extração foi realizada pela ferramenta API de Streaming de mídia social do Twitter, ao todo a base possui 20 mil tweets, foram separados 8.000 tweets para serem rotulados em crime e 12.000 tweets para serem rotulados em não crime, servindo como base para o aprendizado. Esse banco de dados rotulado e em português brasileiro serve como base para o treinamento e aprendizado de classificador. A Tabela 1 mostra um exemplo do banco de dados rotulado, cada linha do arquivo é composta por duas seções: a primeira contendo o texto presente no tweet e a outra indicando respectivamente a presença ou ausência de crime.

Tabela 1. Exemplos de Tweets rotulados como crime e não-crime para treinamento do classificador

\begin{tabular}{ll}
\hline Tweets & Classe \\
\hline Uma mulher é encontrada morta dentro de um cemitério & Crime \\
Ladrões atacam ALEPA para roubar cabos de cobre & Crime \\
Os restos mortais de quatro pessoas são encontrados & Crime \\
Homem preso sob acusação de estuprar criança & Crime \\
Fernanda Gentile passa muito tempo na Rússia: é tranquilo & não-crime \\
20 anos pós derrota, a equipe de 98 finalmente se vingou & não-crime \\
Dol quer saber: Você acha que espancar educa? & não-crime \\
Ex-BBB Marcos Harter será candidato a deputado federal & não-crime
\end{tabular}

Fonte: COSTA, J. P. \& Tal., 2019

Para continuidade do mesmo foi preciso realizar o processo de pré-processamento da base que é uma etapa importante na organização e limpeza de uma base de dados. Os seguintes passos foram executados:

- Conversão de todos os caracteres da base para minúsculo. Dessa forma o mesmo termo escrito de diferentes formas é avaliado como um único termo;

- Remoção das URLs nas mensagens. Estes termos não influenciam na opinião ou polaridade da mensagem;

- Remoção de letras duplicadas. Por exemplo: "muiotooo bom o jogo" é transformado em "muito bom o jogo" para que ambas as frases sejam avaliadas como um único termo;

- Remoção de acentos, pontuação, caracteres especiais e emoticons;

- Remoção de stopwords; e

- Remoção de menções a usuários e hashtags.

Os métodos de word embeddings são uteis para generalização de textos não estruturados e usados em análise de sentimentos. É um conjunto de técnicas ou métodos para mapear a semântica e a sintática de uma linguagem natural com base na estatística (Carvalho, 2018). Estes métodos fornecem boas representações vetoriais contínuas de baixa dimensão para conjunto de textos não estruturados. Esses vetores são muito úteis, porque possuem um forte poder de generalização. Para o objetivo de padronizar diferentes flexões de palavras relacionadas em uma palavra base ou raiz foi utilizado o vetor de palavras BOW (Bag of Words) para o classificador SVM.

\section{RESULTADO}

Um experimento foi realizado com um conjunto de 1.137 textos previamente rotulados e desbalanceados. Para treinar e testar o classificador SVM. O conjunto é composto por frases relacionadas a crime e as que relatam crime. O resultado obtido neste experimento, utilizando SVM com kernel Linear e RBF são apresentados na Tabela 2.

As medidas de avaliação utilizadas no experimento foram Precision (equação 1), Recall (equação 2) e F-measure (equação 3) e são mostradas na sequência. 


$$
\begin{gathered}
\text { Precision }=\frac{V P}{(V P+F P)} \\
\text { Recall }=\frac{V P}{(V P+F N)} \\
F-\text { measure }=\frac{2 . \text { Precision.Recall }}{(\text { Precision }+ \text { Recall })}
\end{gathered}
$$

Onde VP são verdadeiros positivos, FP são falsos positivos e FN são falsos negativos.

O modelo proposto é tarefa de classificação binária, no qual esse modelo computacional deve fornecer duas classes 1 (crime) e 0 (não crime), na Tabela 2, mostramos os resultados das métricas para cada modelo do SVM.

Tabela 2. Resultados Obtidos com o SVM (Kernel Linear)

\begin{tabular}{cccc}
\hline & Precision & Recall & F-Measure \\
\hline SVM (Linear) & & & \\
1 & $\mathbf{0 . 9 7}$ & 0.94 & 0.95 \\
0 & 0.69 & $\mathbf{0 . 8 1}$ & $\mathbf{0 . 7 4}$ \\
SVM (RBF) & & & \\
1 & 0.96 & 0.94 & 0.95 \\
0 & 0.69 & 0.77 & 0.72
\end{tabular}

Analisando os dados obtidos foi obtido uma precisão de 97\% no SVM Linear.

Além das métricas da tabela 2, aplicamos também o método de avaliação denominado curva ROC (AUC). Um AUC mais alto significa que o modelo deu uma probabilidade maior de acertar uma classificação. As instâncias que possuem probabilidade de predição superior a 0,5 de serem classificadas como classe 1 (crime) serão classificadas como tal, caso contrário, serão classificadas como classe 0 (não crime).

A Figura 2 mostra o conjunto de gráficos da curva ROC de cada um dos dois SVM. O SVM Linear e o SVM BRF mostram que a curva está sempre acima de 0,5 da diagonal, isso nos mostra um bom desempenho na eficácia em classificar corretamente, onde valores maiores de AUC indicam melhores resultados e vice-versa.

Analisando o gráfico, notamos que o SVM Linear apresenta uma curva com melhor desempenho que o SVM RBF. A linha de atuação do Linear tende a se aproximar do valor 1.

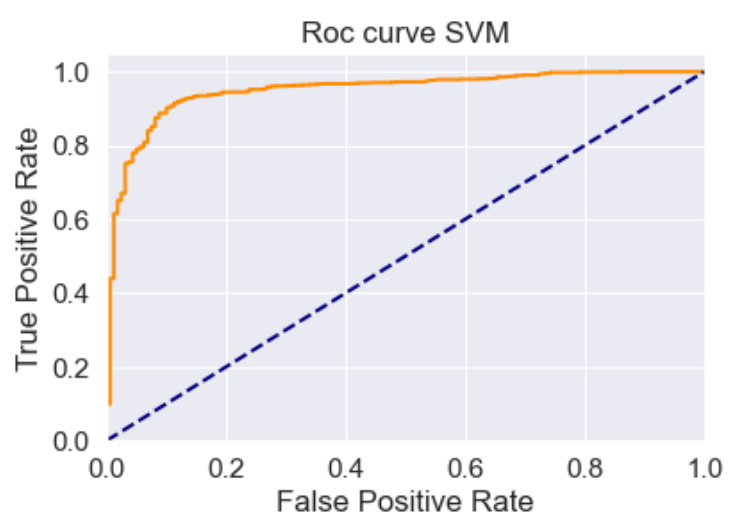

(A)

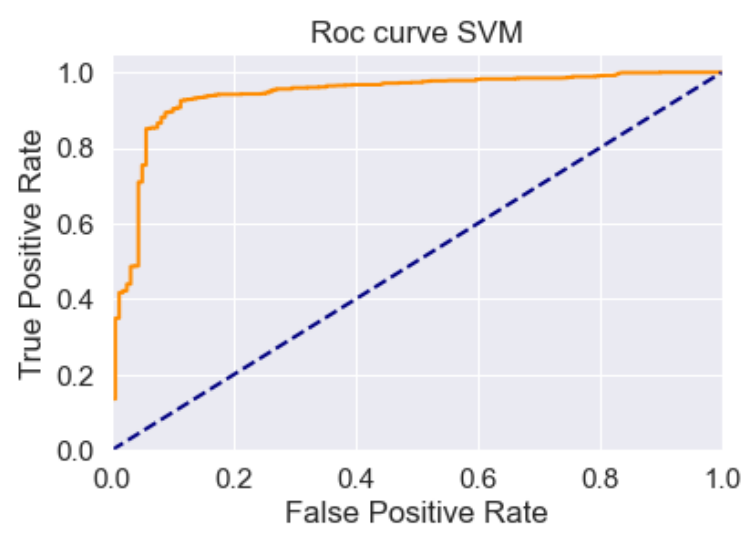

(B)

Figura 2. Gráfico da curva ROC do SVM Linear (A) e SVM RBF (B)

Fonte: Autor, 2020 


\section{CONCLUSÃO}

Nessa pesquisa foi desenvolvido e analisada a aplicação do SVM para a categorização de tweets relacionados a crime ou não crime da cidade de Belém. Assim, foi realizada a comparação dos resultados obtidos com o classificador SVM Linear e o RBF, sendo que os resultados obtidos indicam que uma função Kernel do tipo Linear é aquela que melhor se adapta para a detecção de sentimentos de crime por meio do método de classificação com um bom grau de precisão.

Além do método de classificação de textos relacionados a crimes em Português do Brasil, uma das principais contribuições deste trabalho é tornar disponível um corpus de textos que poderá ser utilizado em outras pesquisas de Classificação e Análise de Sentimento utilizando tweets relacionados a crimes, podendo posteriormente aplicar técnicas que possa realizar a comparação com dados reais para realizar uma comparação se todos os crimes são realizados boletins de ocorrência, ou possivelmente que a partir dos tweets classificados em crimes possam servir como uma fonte de dados estatística para traçar um panorama da violência em bairros, cidades, estados ou até no país.

Como trabalhos futuros, pretende-se melhorar os resultados obtidos neste artigo ampliando-se o número de textos do dataset e pretende-se utilizar outros algoritmos para realizar a comparação com outros classificadores.

\section{AGRADECIMENTOS}

Universidade Federal do Pará (UFPA).

\section{REFERÊNCIAS}

Alwakid, G., Osmam, T., \& Hughes-Roberts, T. (2017). Challenges in sentiment analysis for Arabic social networks. Procedia Computer Science, 117, 89-100.

Awan, I. (2016). Islamophobia on Social Media: A Qualitative Analysis of the Facebook's Walls of Hate. International Journal of Cyber Criminology. 10(1), 1-20.

Bothos, E.; Apostolou, D.; Mentzas, G. Using Social Media to Predict FutureEvents with Agent-Based Markets.IEEE Intelligent Systems, v. 25, n. 6, p. 50Ü58, 2010.

Cerqueira, D., Bueno, S., Lima, R. S. D., Cristina, N., Helder, F., Paloma Palmieri, A., ... \&amp; Pacheco, D. (2019). Atlas of violence 2019 .

Corrêa, T. Igor (2017) Analise de sentimentos expressos na rede social Twitter em relação aos filmes indicados ao Oscar 2017. (Master's dissertation, Federal University of Uberlândia).

Costa, J. P. ; Santos, A. M. T. B. ; Da Mata, Eulália C. ; Da Silva, Marcelino Silva ; Aranha, P. S. ; Francês, C.R.L. . Deep Learning: Application Of The Lstm Model In The Categorization Of Tweets On Violence In The City Of Belém. In: $4^{a}$ Conferência Internacional sobre Big Data Analytics, Data Mining e Inteligência Computacional, 2019, Porto. Big Data Analytics, Data Mining And Computational Intelligence 2019 And Theory And Practice In Modern Computing 2019, 2019. v. 1. p. 54-60.

Filho, J. A. C. Mineração De Textos: Análise de Sentimento Utilizando Tweets Referentes à Copa Do Mundo 2014.

Folha, S. P. Twitter registra crescimento em usuários diários e nas receitas. 2019. Disponível em:<Acesso em: 04 Setembro, 2020>.

Irfan, R. et al. A survey on text mining in social networks. The Knowledge Engineering Review, [S.1.], v. 30, n. 2, p. 157 -170, mar. 2015. Disponível em:<http://dx.doi.org/10.1017/S0269888914000277>. Acesso em: 13 Setembro. 2020.

Java, A., Song, X., Finin, T., and Tseng, B. (2007). Why we twitter: understanding microblogging usage and communities. In Proceedings of the 9th Web KDD and 1st SNA-KDD 2007 workshop on Web mining and social network analysis, pages 56-65. ACM.

Junior Gilson. Medeiros de Oliveira. TCC. Máquina de Vetores Suporte: estudo e análise de parâmetros para otimização de resultado. UFPE 2010. < https://www.cin.ufpe.br/ tg/2010-2/gmoj.pdf> Acesso: 24/agosto 2020.

Kapko, M. Twitter's impact on 2016 presidential election is unmistakable. 2016. Disponível em:<Acesso em: 04 Setembro, 2020>. 
Lorena, A. C. de Carvalho, A. C. P. L. F. (2003) Introdução às Máquinas de Vetores Suporte (Support Vector Machines). Relatório Técnico $n^{\circ} 192$ do Instituto de Ciências Matemáticas e de Computação da USP.

Manning, C. D.Raghavan, P. Schütze, H. (2008) Introduction to Information Retrieval, Cambridge University Press.

Massuchin, M. G., \&amp; de Sousa, S. G. (2020). From print to fanpages: language and format of Brazilian regional newspapers\&\#39; content on Facebook from a comparative perspective. Observatories (OBS*), 14(1).

Nayak, A.S. et al. Surveyon Pre-Processing Techniques for Text Mining. International Journal of Engineering and Computer Science, [S.1.], v.5, p.16875-16879, jun.2016. Disponível em: <http://ijecs.in/index.php/ijecs/article/download/2019/1868>. Acesso em: 21 setembro, 2020.

Oliveira. Larissa Lages de. TCC. Uma Análise de Algoritmos de Aprendizagem de Máquina Aplicados em Técnicas de Localização Indoor para Diferentes Tipos de Smartphones. UFPE. Recife, Julho de 2017. < https://www.cin.ufpe.br/ tg/2017-1/llo_tg.pdf> Acesso: 20/Setembro 2020.

Pang, B. Lee, L. Vaithyanathan, S. (2002) Thumbs up? Sentiment Classification Using Machine Learning Techniques. In: Proceedings of the Conference on Empirical Methods in Natural Language Processing (EMNLP-2002). Philadelphia, Pennsylvania, pp. 79-86.

Romi, Fátima Auxiliadora Bezerra Lima. Análise das redes sociais informais com foco no crescimento profissional das pessoas: um estudo de caso / Fátima Auxiliadora Bezerra Lima Romi. - Niterói, 2013. 92 f Disponível em: < https://app.uff.br/riuff/bitstream/1/4430/1/Dissert\%20Fatima\%20\%20Auxiliadora\%20B\%20L\%20\%20Romi.pdf>. Acesso em: 10 Outubro, 2020.

Sebastiani, F. "Machine learning in Automated Text Categorization", Technical report, IEI B4-31-12-99, Instituto di Elaborazione della Informazione, Consiglio Nazionale delle Ricerche, 1999

Smola, A. J. Barlett, P. Schölkopf, B. Schuurmans, D. (1999) Introduction to Large Margin Classifiers. MIT Press. Carvalho, C. M. A. (2018). Comparative study of sentiment analysis applied to public news. ((Master\&\#39;s dissertation, Federal University of Maranhão)

Vapnik, V., "The Nature of Statistical Learning Theory", Springer 1995

Vilela. Paula. Dissertação. $4-$ Support Vector Machines. PUC - Rio- Certicação Digital No 0912855/CA.<http://www2.dbd.puc-rio.br/pergamum/tesesabertas/0912855_2011_cap_4.pdf > Acesso: 23/agosto 2020.

Witten, I.H., Frank E., Hall, M.A.(2011) Data Mining: Practical Machine Learning Tools and Techniques. San Francisco: Morgan Kaufmann, 3 ed.

Wunnasri, Theeramunkong e Haruechaiyasak, (2013). Resolvendo dados desequilibrados para análise de sentimento tailandês. Conferencia Internacional em Ciências da Computação e Engenharia de Software, 200 - 205.

Zanchini. Vinícius Augusto Alves. Criação de um modelo de classificação de tweets depressivos utilizando máquina de vetores de suporte. Universidade Federal de Uberlândia 2019.< https://repositorio.ufu.br/bitstream/123456789/26342/4/Cria\%C3\%A7\%C3\%A3oModeloClassifica\%C3\%A7\%C3\% A3o.pdf > Acesso: 23/Setembro 2020. 